\title{
Value of PAX1 Methylation Analysis by MS-HRM in the Triage of Atypical Squamous Cells of Undetermined Significance
}

\author{
Shi-Rong Li ${ }^{1}$, Zhen-Ming Wang ${ }^{1 *}$, Yu-Hui Wang${ }^{1}$, Xi-Bo Wang ${ }^{2}$, Jian-Qiang \\ Zhao $^{1}$, Hai-Bin Xue ${ }^{1}$, Fu-Guo Jiang ${ }^{1}$
}

\begin{abstract}
Background: Detection of cervical high grade lesions in patients with atypical squamous cells of undetermined significance (ASCUS) is still a challenge. Our study tested the efficacy of the paired boxed gene 1 (PAX1) methylation analysis by methylation-sensitive high-resolution melting (MS-HRM) in the detection of high grade lesions in ASCUS and compared performance with the hybrid capture 2 (HC2) human papillomavirus (HPV) test. Materials and Methods: A total of 463 consecutive ASCUS women from primary screening were selected. Their cervical scrapings were collected and assessed by PAX1 methylation analysis (MS-HRM) and high-risk HPV-DNA test (HC2). All patients with ASCUS were admitted to colposcopy and cervical biopsies. The Chisquare test was used to test the differences of PAX1 methylation or HPV infection between groups. Results: The specificity, sensitivity, and accuracy for detecting CIN2 + lesions were: $95.6 \%, 82.4 \%$, and 94.6\%, respectively, for the PAX1 MS-HRM test; and 59.7\%, 64.7\%, and 60.0\% for the HC2 HPV test. Conclusions: The PAX1 methylation analysis by MS-HRM demonstrated a better performance than the high-risk HPV-DNA test for the detection of high grade lesions (CIN2 +) in ASCUS cases. This approach could screen out the majority of low grade cases of ASCUS, and thus reduce the referral rate to colposcopy.
\end{abstract}

Keywords: ASCUS - PAX1 - methylation - MS-HRM analysis - HPV

Asian Pac J Cancer Prev, 16 (14), 5843-5846

\section{Introduction}

Optimal treatment option for women with ASCUS is not well established. Most low-grade lesions regress spontaneously. On the other hand, a small but significant proportion of cases with ASCUS may harbour cervical intraepithelial neoplasia (CIN)2-3, or even invasive carcinoma (Arbyn et al., 2004; Yarandi et al., 2009). Persistent infection with high-risk HPV (HR-HPV) is the most important etiologic factor for cervical cancer. In almost every invasive cervical cancer patient, HPV can be recognized (Park et al., 1996; Wang et al., 2013; Siriaunkgul et al., 2014). In consequence, HR-HPV testing has been widely used for the triage of ASCUS patients (Wentzensen et al., 2010). Although HR-HPV testing has a good sensitivity , but the high prevalence of transient human papillomavirus infections limits it's specificity (Dehn et al., 2007). Thus, there is a need for other markers which have high sensitivity and specificity. Recently, paired boxed gene 1 (PAX1) was reported as a new methylation-silenced gene in cervical cancer (Lai et al., 2008). Recently, methylationsensitive high-resolution melting (MS-HRM) analysis has been introduced as a sensitive and rapid technique for the quantification of DNA methylation (Wu et al., 2011; Dimitrakopoulos et al., 2012).
In our study, we tested the efficacy of PAX1 methylation analysis by MS-HRM for the triage of patients with ASCUS, and compared its performance with HRHPV testing.

\section{Materials and Methods}

\section{Patient samples}

A total of 463 consecutive patients (mean age 37 years, range 20-58) who participated in the cervical cytological screening by Thinprep cytologic test (TCT) technique in the Weifang city people's hospital from January 2013 to December 2014, and with an ASCUS diagnosis based on the cytological screening, were selected. The cytological screening was performed using The 2001 Bethesda System (TBS). The exclusion criteria included current pregnancy, history of cancer at other sites, and history of immune compromise diseases. The cervical scrapings of 463 ASC-US cases were collected and evaluated by using PAX1 methylation analysis (MS-HRM) and high-risk HPV-DNA test (HC2). All patients with ASCUS were admitted for colposcopy and cervical biopsy. Cervical biopsies were cut and stained with hematoxylin and eosin, read by a pathologist, and confirmed by a second independent reading result. 
HPV test

High-risk HPV-DNA (HR-HPV) infection of the cervical sample was detected by the HC2 test ((Digene Corp., Silver Spring, MD, USA). The specimen DNA was denatured and hybridized with the RNA probes which directed against 13 high-risk human papillomavirus. The hybrids were captured by hybrid-specific antibodies and detected by second antibody and chemiluminescence.

\section{MS-HRM analysis of PAXI}

DNA of cervical scrapings was extracted using the QIAamp DNA mini kit (Qiagen) and bisulfite converted using the EpiTect Bisulfite Kit (Qiagen) according to the instructions of manufacturer. The bisulfite converted DNA was PCR-amplified with the specific primers (forward, 5'-CGGGAATTAATGAGTTGTTAATT-3'; reverse, 5'-AAACCCAAAATAAACTTCATCC-3') in a HRM Epigenetics PCR Kit (Qiagen). DNA extracted from normal human peripheral blood leukocytes was used as unmethylated control DNA. DNA of human peripheral blood leukocytes treated with M.SssI methyltransferase served as the fully methylated control. Standards of methylation were constructed by diluting $100 \%$ methylated bisulfite-modified control DNA in a pool of normal bisulfite-modified DNA at ratios of 10 , $30,50,80$ and $100 \%$. MS-HRM was conducted in a $25 \mu \mathrm{l}$ volume containing $2 \mu \mathrm{l}$ of modified template DNA, $12.5 \mu \mathrm{l}$ $2 \times$ EpiTect HRM PCR Master Mix (HRM Epigenetics PCR Kit, Qiagen), $1.9 \mu$ l of each primer $(10 \mu \mathrm{M})$, and $6.7 \mu$ of PCR grade water. The PCR was subjected to an incubation at $95^{\circ} \mathrm{C}$ for 10 minutes, followed by 40 cycles of $95^{\circ} \mathrm{C}$ for $10 \mathrm{~s}$, annealing for $10 \mathrm{~s}$ from $62^{\circ} \mathrm{C}$ to $50^{\circ} \mathrm{C}$ (decrease $0.5^{\circ} \mathrm{Cper}$ cycle), extension at $72^{\circ} \mathrm{C}$ for $10 \mathrm{~s}$; followed by an $\mathrm{HRM}$ of $95^{\circ} \mathrm{C}$ for $1 \mathrm{~min}$, and $40^{\circ} \mathrm{C}$ for 1 $\min , 65^{\circ} \mathrm{C}$ for $15 \mathrm{~s}$, and continuous acquisition to $95^{\circ} \mathrm{C}$ at one acquisition per $0.02^{\circ} \mathrm{C}$, and then detected using the Roche LightCycler (Roche). HRM data were analyzed by use of the dedicated HRM software (LightCycler 480 Gene Scanning Software). All experiments were performed in duplicate. This study was approved by the institutional review board of the Weifang city people's hospital in Shan dong province, PRC, and informed consents were obtained from all participants.

\section{Statistical analysis}

SPSS 21.0 software was used for statistical analyses. Chi-square test was used to test the differences of PAX1 methylation or HPV infection between groups. $p$ values less than 0.05 was considered statistically significant. Using PAX1 methylation and high-risk HPV test results make receiver operating characteristic curve, calculating the area under the curve.

\section{Results}

\section{The sensitivity of the PAX1 MS-HRM assay}

The sensitivity of the PAX1 MS-HRM was assessed by using the consistency of normalized melting profiles derived from samples with different ratios of methylated template: 10, 30, 50, 80 and $100 \%$ methylated. PAX1 MS-HRM was able to reproducibly detect $10 \%$ methylated
DNA in a background of unmethylated DNA. At a cutoff value of $10 \%$, In 47 out of 463 ASCUS scrapings, PAX1 methylation was identified. Figure 1 represents the PAX1 MS-HRM results from two representative samples.

The status of PAX1 methylation and HR-HPV infection in Patients With ASCUS

Quantitative assessment of PAX1 methylation by MSHRM according to disease severity is shown in Table 1. At the cutoff value of $10 \%, 100 \%$ of the cervical carcinoma, $92.3 \%$ of the CIN3 and $73.7 \%$ of CIN2 specimens were positive for PAX1 methylation compared with $0 \%$ for the inflammation, $8.2 \%$ for CIN1 specimens (Table 1). At this cut off value, specimens positive for CIN2 + were more significant than CIN2 - specimens $(\mathrm{P}<0.001)$. As a comparison, the HC2 HPV test result was positive in $100 \%$ of the cervical carcinoma, $69.2 \%$ of the CIN3 and $57.9 \%$ of CIN2 specimens. Noteworthily, HPV was present in $60.2 \%$ and $17.2 \%$ of the benign conditions of CIN1 and the inflammation, respectively, but PAX1 methylation was present in only $8.2 \%$ and $0 \%$, respectively (Table 1 ).

\section{The detection efficiency of PAX1 methylation and HR-HPV infection for CIN2+}

We compared the performance of MS-HRM for PAX1 methylation detecting to the HR-HPV test in the detection of CIN2 + by receiver operating characteristic curve (Figure 2). The area under the curve of PAX1 methylation and HR-HPV test were 0.890 and $0.622(p<0.05)$. The specificity and sensitivity of PAX1 MS-HRM assay were

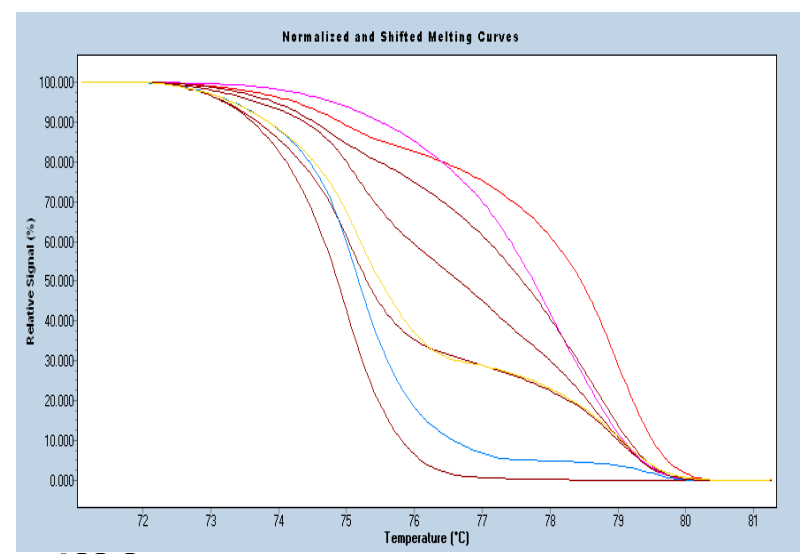

Figure 1. PAX1 MS-HRM Curves Representing the Methylation Level of Two Samples. (From left to right , the curve representing the methylation status:0, $10 \%$, sample1, $30 \%, 50 \%, 80 \%$, sample $2,100 \%$ )

Table 1. The Status of PAX1 Methylation and HR-HPV Infection in Patients With ASCU

\begin{tabular}{lrrr}
\hline Histopathology & $\mathrm{n}$ & PAX1 MS-HRM & \multicolumn{1}{c}{ HR-HPV } \\
\hline inflammation & 198 & $0.0 \%(0 / 198)$ & $17.2 \%(34 / 198)$ \\
CIN1 & 231 & $8.2 \%(19 / 231)$ & $60.2 \%(139 / 231)$ \\
CIN2 & 19 & $73.7 \%(14 / 19)$ & $57.9 \%(11 / 19)$ \\
CIN3 & 13 & $92.3 \%(12 / 13)$ & $69.2 \%(9 / 13)$ \\
cervical carcinoma 2 & $100 \%(2 / 2)$ & $100 \%(2 / 2)$ \\
CIN2 + & 34 & $82.4 \%(28 / 34)^{* a}$ & $64.7 \%(22 / 34) * b$ \\
CIN2 - & 429 & $4.4 \%(19 / 429)$ & $40.3 \%(173 / 429)$ \\
\multicolumn{2}{l}{ (CIN1 or inflammation) } & &
\end{tabular}

*comparing CIN2 + group with CIN2 - group, ${ }^{a} \mathrm{P}=0.0000 ;{ }^{b} \mathrm{P}=0.0056$ 


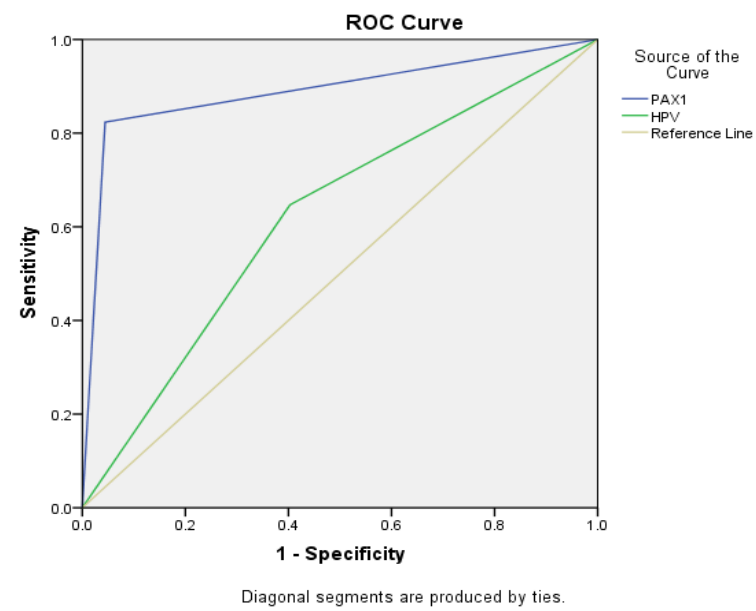

Figure 2. The Receiver Operating Characteristic Curve of PAX1 Methylation and HR-HPV Test

$95.6 \%$ and $82.4 \%$, compared with $59.7 \%$ and $64.7 \%$, respectively, for detection by HR-HPV test. The negative predictive value (NPV), positive predictive value (PPV), and accuracy of PAX1 MS-HRM assay are all superior to those of the HR-HPV test. The NPV, PPV, and accuracy for detecting CIN2 + lesions were $98.6 \%, 59.6 \%$, and $94.6 \%$, respectively, for PAX1 MS-HRM test; $95.5 \%, 11.3 \%$, and $60.0 \%$, respectively, for HR-HPV test.

\section{Discussion}

Organized cytological screening resulted in a significant decrease in the proportion of women with cervical cancer, and accordingly, the number of ASCUS diagnoses has a marked increase. A significant number of cervical cancers occur after a normal cervical smear following ASCUS (Andrae et al., 1999). In this study, we screens out 34 high grade lesions cases in 463 women with ASCUS. A important problem of cytology screening is the limited sensitivity (Andersson et al., 2005; Naucler et al., 2009). Therefore, if a first cytology screening shows a diagnosis with ASCUS, a second normal cervical smear is not suitable for the exclusion of precancerous cervical lesion. Another strategy is to refer these patients to colposcopy-directed biopsy, but it is costly and invasive.

Adding the HR-HPV test is a proposed method to secondary cytology screening. Triage study have shown that HPV test is more sensitive than repeated cytology smear for determining high grade lesions in women with ASCUS (Silverloo et al., 2009). But the low PPV (37\%) and specificity (31\%)even lead to a worse situation, because they make more patients undergoing unnecessary referrals (Nieh et al., 2005).

This study demonstrates that MS-HRM for PAX1 methylation test is better than $\mathrm{HC} 2 \mathrm{HPV}$ test in the triage of ASCUS. Indeed, it is the most difficult for pathologists to reproduce among all CIN2 diagnosis, because the diagnosis of CIN2 has been a gray area (Carreon et al., 2007). For a long time, CIN2 was considered an intermediate entity that may be under called as CIN1 or over called as CIN3. In the detection of CIN2 +, the specificity and sensitivity of PAX1 MS-HRM assay were $95.6 \%$ and $82.4 \%$, compared with $59.7 \%$ and $64.7 \%$, for detection by HPV test. The NPV, PPV , and accuracy of PAX1 MS-HRM assay are all superior to those of the HC2 HR-HPV test. The MS-HRM for PAX1 methylation testing can screens out the majority high grade lesions cases of ASCUS , and may avoid unnecessary invasive inspection. Because of the influence of prevalence rate, the PPV of MS-HRM for PAX1 methylation testing is not high in this study.

In 2007, MS-HRM analysis was first described by Wojdacz et al for high throughput and sensitive assessment of DNA methylation (Wojdacz et al., 2007). The PCR amplification products generated from bisulfite- modified DNA templates with different contents of methylcytosine show differences in melting temperature, which can be analyzed by a fluorometer thermal cycler. Quantification was implemented by a standard curve generated with serial dilutions of methylated DNA. MS-HRM assay has important advantage over the Methylation-Specific PCR. MS-HRM can scan all of the CpGs in the target sequence. It also resolves heterogeneous methylation (Candiloro et al., 2011), allows gene amplification and methylation analysis in one closed tube. Closed-tube approaches have important advantage, as they minimize the risk of cross contamination. Moreover, MS-HRM experiment doesn't require a reference assay for normalization. A new Quantitative Methylation-Specific PCR (QMSP) method was used to analyze the gene methylation, but it require a reference assay and expensive fluorescence probe (Huang et al., 2010). All of these advantages make MS-HRM assay rapid, reliable and cost-effective (Wojdacz et al., 2012).

In summary, the MS-HRM for PAX1 methylation testing have a better performance than HR-HPV test in the detection of high grade lesions CIN2 + in ASCUS. This approach could screens out the majority high grade lesions cases of ASCUS, also could reduce the referral rate to colposcopy. Our results may provide a new triage method for women with ASCUS. Because the number of cases particulatrly in the CIN3 and carcinoma groups is small, further studies in larger screening populations are warranted to prove its efficacy in screening ASCUS.

\section{Acknowledgements}

This study was supported by grants from the Weifang Municipal Science and Technology Development Program (No. 201302014).

\section{References}

Andersson S, Dillner L, Elfgren K, et al (2005). A comparison of the human papillomavirus test and Papanicolaou smear as a second screening method for women with minor cytological abnormalities. Acta Obstet Gynecol Scand, 84, 996-1000.

Andrae B, Smith P (1999). Clinical impact of quality assurance in an organized cervical screening program. Acta Obstet Gynecol Scand, 78, 429-35.

Arbyn M, Buntinx F, Van Ranst M, et al (2004). Virologic versus cytologic triage of women with equivocal Pap smears: a meta-analysis of the accuracy to detect high-grade intraepithelial neoplasia. J Natl Cancer Inst, 96, 280-93.

Candiloro IL, Mikeska T, Dobrovic A (2011). Assessing combined methylation-sensitive high resolution melting 
and pyrosequencing for the analysis of heterogeneous DNA methylation. Epigenetics, 6, 500-7.

Carreon JD, Sherman ME, Guillen D, et al (2007). CIN2 is a much less reproducible and less valid diagnosis than CIN3: results from a histological review of population-based cervical samples. Int J Gynecol Pathol, 26, 441-6.

Dehn D, Torkko KC, Shroyer KR (2007). Human papillomavirus testing and molecular markers of cervical dysplasia and carcinoma. Cancer, 111, 1-14.

Dimitrakopoulos L, Vorkas PA, Georgoulias V, Lianidou ES (2012). A closed-tube methylation-sensitive high resolution melting assay (MS-HRMA) for the semi-quantitative determination of CST6 promoter methylation in clinical samples. BMC Cancer, 12, 486.

Huang TH, Lai HC, Liu HW, et al (2010). Quantitative analysis of methylation status of the PAX1 gene for detection of cervical cancer. Int J Gynecol Cancer, 20, 513-9.

Lai HC, Lin YW, Huang TH, et al (2008). Identification of novel DNA methylation markers in cervical cancer. Int J Cancer, 123, 161-7.

Naucler P, Ryd W, Tornberg S, et al (2009). Efficacy of HPV DNA testing with cytology triage and/or repeat HPV DNA testing in primary cervical cancer screening. J Natl Cancer Inst, 101, 88-99.

Nieh S, Chen SF, Chu TY, et al (2005). Is p16 (INK4A) expression more useful than human papillomavirus test to determine the outcome of atypical squamous cells of undetermined significance-categorized Pap smear? A comparative analysis using abnormal cervical smears with follow-up biopsies. Gynecol Oncol, 97, 35-40.

Park JS (1996). The role of HPV DNA testing in cervical neoplasia. J Obstet Gynaecol Res, 22, 611-20.

Silverloo I, Andrae B, Wilander E (2009). Value of high-risk HPV-DNA testing in the triage of ASCUS. Acta Obstet Gynecol Scand, 88, 1006-10.

Siriaunkgul S, Settakorn J, Sukpan K, et al (2014). Populationbased cervical cancer screening using high-risk HPV DNA test and liquid-based cytology in northern Thailand. Asian Pac J Cancer Prev, 15, 6837-42.

Wang JL, Yang YZ, Dong WW, et al (2013). Application of human papillomavirus in screening for cervical cancer and precancerous lesions. Asian Pac J Cancer Prev, 14, 2979-82.

Wentzensen N, Wilson LE, Wheeler CM, et al (2010). Hierarchical clustering of human papilloma virus genotype patterns in the ASCUS-LSIL triage study. Cancer Res, 70, 8578-86.

Wojdacz TK, Dobrovic A (2007). Methylation-sensitive high resolution melting (MS-HRM): a new approach for sensitive and high-throughput assessment of methylation. Nucleic Acids Res, 35, 41.

Wojdacz TK (2012). Methylation-sensitive high-resolution melting in the context of legislative requirements for validation of analytical procedures for diagnostic applications. Expert Rev Mol Diagn, 12, 39-47.

Wu W, Zhang J, Yang H, Shao Y, Yu B (2011). Examination of AKAP12 promoter methylation in skin cancer using methylation-sensitive high-resolution melting analysis. Clin Exp Dermatol, 36, 381-5.

Yarandi F, Shojaei H, Eftekhar Z, Izadi-Mood N (2009). Comparison of three management strategies for patients with atypical squamous cells of undetermined significance, after six months delay: a three-year experience in an Iranian university hospital. Aust NZJ Obstet Gynaecol, 49, 207-10. 